\title{
How to Build Input/Output Logic
}

\author{
Xin Sun \\ Individual and Collective Reasoning Group, University of Luxembourg
}

\begin{abstract}
In this paper we anatomize input/output logic. We analyze various derivation rules in isolation and define the corresponding semantics. We thus create a toolbox to build input/output logic. We use this toolbox to correct a mistake appeared in the work of applying input/output logic to constitutive norms. We further develop fixed point characterizations for input/output logic involving rules of cumulative transitivity and present new completeness proofs.
\end{abstract}

Key words: input/ouput logic, fixed point, deontic logic

\section{Introduction}

In the first volume of the handbook of deontic logic [10], input/output logic [1820,22] appears as one of the new achievement in deontic logic in this century. Input/output logic takes its origin in the study of conditional norms. Unlike the modal logic framework [29], which usually uses possible world semantics, input/output logic adopts mainly operational semantics: a normative system is conceived in input/output logic as a deductive machine, like a black box which produces normative statement as output, when we feed it descriptive statements as input.

Such an operational treatment can be traced back to Alchourron and Bulygin [1]. Boella and van der Torre [3] extended input/output logic to reasoning about constitutive norms. Tosatto et al. [8] adapted it to represent and reason about abstract normative systems. For a comprehensive introduction of input/output logic, see Parent and van der Torre [22].

The procedure of operational semantics is divided to three stages. In the first stage, we have in hand a set of propositions (call it the input) as a description of the current state. We can then apply logical operators to this set, say close the set by logical consequence. Then we pass this set to the deductive machine and we reach the second stage. In the second stage, the machine accepts the input and produces a set of propositions as output. In the third stage, we accept the output and apply logical operators to it.

On the proof-theoretical side, input/output logics are characterized by derivation rules about norms, which is represented by an ordered pair of formulas. Given a set of norms $N$, a derivation system is the smallest set of norms which contains $N$ and is closed under certain derivation rules.

One feature of the existing work of input/output logic is: the derivation rules always work in bundles. For example in simple-minded input/output logic of Makinson and van der Torre [18], the derivation system is decided by three rules: strengthening the input (SI), weakening the output (WO) and conjunction in the output (AND). When 
several derivation rules work together, the corresponding operational semantics will be rather complex, and insights of the machinery will therefore be concealed. To achieve a deeper understanding on input/output logic, it is helpful to isolate every single rule and study them separately. This is the motivation of this paper.

In this paper we anatomize input/output logic. We take a close look at various rules in isolation and define the corresponding semantics. Not surprisingly, as long as we have semantics for single rule, we can use it as a toolbox to construct semantics for systems decided by multiple rules.

The structure of this paper is as following: we first review input/output logic in Section 2. Then we study a number of rules from Section 3 to 6. In Section 7 we use the result of this paper to correct a mistake of Boella and van der Torre [3]. We then discuss related work in Section 8. We conclude this paper with future work in Section 9. For the sake of readability, all complex proof are given in the appendix.

\section{Background}

In this section we review input/output logic. Let $\mathbb{P}=\left\{p_{0}, p_{1}, \ldots\right\}$ be a countable set of propositional letters and $L$ be the propositional language built upon $\mathbb{P}$. Let $N \subseteq L \times L$ be a set of ordered pairs of formulas of $L$. We call $N$ a normative system. A pair $(a, x) \in N$, call it a norm, is read as "given $a$, it ought to be $x$ ". $N$ can be viewed as a function from $2^{L}$ to $2^{L}$ such that for a set $A$ of formulas, $N(A)=\{x:(a, x) \in N$ for some $a \in A\}$.

Makison and van der Torre define the operations from out $t_{1}$ to $\mathrm{out}_{4}$ as follows:

$$
\begin{aligned}
& \text { - } \text { out }_{1}(N, A)=C n(N(C n(A))) \\
& \text { - out }(N, A)=\bigcap\{C n(N(V)): A \subset V, V \text { is complete }\} \\
& \text { - } \text { out }_{3}(N, A)=\bigcap\{C n(N(B)): A \subseteq B=C n(B) \supseteq N(B)\} \\
& \text { - out } 4(N, A)=\bigcap\{C n(N(V): A \subseteq V \supseteq N(V)), V \text { is complete }\}
\end{aligned}
$$

Here $C n$ is the classical consequence operator of propositional logic, and a set of formulas is complete if it is either maxi-consistent or equal to $L$. For each of these four operations, a throughput version that allows inputs to reappear as outputs is defined as out $_{n}^{+}(N, A)=\operatorname{out}\left(N_{i d}, A\right)$, where $N_{i d}=N \cup\{(a, a) \mid a \in L\}$.

Input/output logics are given a proof theoretic characterization. We say that an ordered pair of formulas is derivable from a set $N$ iff $(a, x)$ is in the least set that includes $N$ and is closed under a number of rules. The following are the rules we use:

- SI (strengthening the input): from $(a, x)$ to $(b, x)$ whenever $b \vdash a$

- WO (weakening the output): from $(a, x)$ to $(a, y)$ whenever $x \vdash y$

- AND (conjunction of output): from $(a, x)$ and $(a, y)$ to $(a, x \wedge y)$

- OR (disjunction of input): from $(a, x)$ and $(b, x)$ to $(a \vee b, x)$

- CT (cumulative transitivity): from $(a, x)$ and $(a \wedge x, y)$ to $(a, y)$

- ID (identity): from nothing to $(a, a)$, for every $a \in L$.

The derivation system based on the rules SI, WO and AND is called deriv $v_{1}$. Adding OR to deriv ${ }_{1}$ gives deriv ${ }_{2}$. Adding CT to deriv ${ }_{1}$ gives deriv ${ }_{3}$. The five rules together 
give deriv $_{4}$. Adding ID to $\operatorname{deriv}_{i}$ gives $\operatorname{deriv}_{i}^{+}$for $i \in\{1, \ldots, 4\}$. We use $(a, x) \in$ $\operatorname{deriv}(N)$ to denote the norm $(a, x)$ is derivable from $N$ using rules of derivation system deriv. In Makinson and van der Torre [18], the following completeness theorems are given:

Theorem 1 ([18]). Given an arbitrary normative system $N$ and a formula a,

- $x \in$ out $_{i}(N, a)$ iff $(a, x) \in \operatorname{deriv}_{i}(N)$, for $i \in\{1,2,3,4\}$

- $x \in$ out $_{i}^{+}(N, a)$ iff $(a, x) \in \operatorname{deriv}_{i}^{+}(N)$, for $i \in\{1,2,3,4\}$

\section{Rules of input}

In this section we investigate the following rules regulating the input:

- input equivalence (IEQ): from $(a, x)$ and $a \dashv \vdash b$ to $(b, x) .{ }^{1}$

- strengthening the input (SI): from $(a, x)$ to $(b, x)$ whenever $b \vdash a$.

- disjunction of input (OR): from $(a, x)$ and $(b, x)$ to $(a \vee b, x)$.

IEQ is a basic rule in the logic of constitutive norms [15]. SI is involved in all input/output logics of Makinson and van der Torre. OR is valid in out 2 and out 4 . OR is in some sense problematic and can cause paradoxes. But it is heavily used in daily life and on the technical side, it is the most interesting rule among those rules of input. The derivation systems decided by rules of input are defined as follows:

Definition 1. Let $D_{i e}(N), D_{s i}(N), D_{o r}(N)$ be the derivation system decided by the rule IEQ, SI, OR respectively. That is, $D_{i e}(N)$ is the smallest set of norms such that $N \subseteq D_{i e}(N)$ and $D_{i e}(N)$ is closed under the IEQ rule, and similarly for $D_{s i}(N)$ and $D_{\text {or }}(N)$.

Now our task is to construct the semantics corresponding to those derivation systems. For the convenience of notation, we let $C_{e}(A)=\{b \in L \mid \exists a \in A, a \dashv \vdash b\}$, for a set $A \subseteq L$. Moreover, we call a set $A$ disjunctive iff it satisfies the following: for all $x \vee y \in A$, either $x \in A$ or $y \in A$. The following is the definition of semantics corresponding to the rules of input.

Definition 2. For a set of norms $N$ and a formula a, we define $O^{i e}(N, a)=N\left(C_{e}(\{a\})\right)$, $O^{s i}(N, a)=N(C n(a)), O^{o r}(N, a)=\bigcap\{N(B) \mid a \in B, B$ is disjunctive $\}$.

\section{Theorem 2.}

1. $(a, x) \in D_{i e}(N)$ iff $x \in O^{i e}(N, a)$.

2. $(a, x) \in D_{s i}(N)$ iff $x \in O^{s i}(N, a)$.

3. $(a, x) \in D_{\text {or }}(N)$ iff $x \in O^{\text {or }}(N, a)$.

Remark 1 The above result reveals that rules of input correspond to operations in the first stage: SI means close the input by logical consequence; IEQ mean close the input by logical equivalence; OR ensures the input has to be extend to satisfy disjunctive property.

\footnotetext{
${ }^{1}$ Here $a \dashv b b$ means $a \vdash b$ and $b \vdash a$
} 


\section{Rules of output}

In this section we investigate the following rules regulating the output:

- output equivalence (OEQ): from $(a, x)$ and $x \dashv \vdash y$ to $(a, y)$.

- weakening the output (WO): from $(a, x)$ to $(a, y)$ whenever $x \vdash y$.

- conjunction of output (AND): from $(a, x)$ and $(a, y)$ to $(a, x \wedge y)$.

OEQ is a basic rule in the of logic constitutive norms [15]. WO and AND are involved in all input/output logics of Makinson and van der Torre. The derivation systems decided by rules of output are defined as follows:

Definition 3. Let $D_{o e}(N), D_{w o}(N), D_{a n}(N)$ be the derivation system decided by the rule OEQ, WO, AND respectively. That is, $D_{o e}(N)$ is the smallest set of norms such that $N \subseteq D_{o e}(N)$ and $D_{o e}(N)$ is closed under the $O E Q$ rule, and similarly for $D_{w o}(N)$ and $D_{a n}(N)$.

For a set of propositional formula $A \subseteq L$, let $C_{s}(A)=\{b \in L \mid \exists a \in A, a \vdash b\}$, $C_{a}(A)=\left\{x \in L\right.$ : there exist $x_{1}, \ldots, x_{n} \in A, x$ is $\left.x_{1} \wedge \ldots \wedge x_{n}\right\}$. The following is the definition of semantics corresponding to the rules of output. For simplicity of notation, $N(a)$ is short for $N(\{a\})$.

Definition 4. For every set of norms $N$ and formula a, we define $O^{o e}(N, a)=C_{e}(N(a))$, $O^{w o}(N, a)=C_{s}(N(a)), O^{a n}(N, a)=C_{a}(N(a))$.

\section{Theorem 3.}

1. $(a, x) \in D_{o e}(N)$ iff $x \in O^{o e}(N, a)$.

2. $(a, x) \in D_{w o}(N)$ iff $x \in O^{w o}(N, a)$.

3. $(a, x) \in D_{a n}(N)$ iff $x \in O^{a n}(N, a)$.

Proof. The prove is straightforward and left to readers.

Remark 2 The above result reveals that rules of output correspond to operations in the third stage: WO means close the input by logical consequence; OEQ means close the input by logical equivalence; AND ensures the output is closed under conjunction.

\section{Rules of normative system}

While rules of input and output affects first stage and third stage respectively, rules of normative system affects the second stage. We investigate three three rules of the normative system:

- zero premise (Z): from nothing to $(T, T)$

- identity (ID): from nothing to $(a, a)$, for every $a \in L$.

- conditioning (CD) from nothing to $(a, b)$, for every $a, b \in L$ such that $a \vdash b$

Definition 5. $D_{z}(N), D_{i d}(N), D_{c d}(N)$ is the derivation system decided by the rule $Z$, ID and CD respectively. 
Definition 6. For every set of norms $N$, let $N_{z}=N \cup\{(\top, \top)\}, N_{i d}=N \cup\{(a, a) \mid$ $a \in L\}, N_{c d}=N \cup\{(a, b) \mid a, b \in L, a \vdash b\}$. We define $O^{z}(N, a)=N_{z}(a)$, $O^{i d}(N, a)=N_{i d}(a), O^{c d}(N, a)=N_{c d}(a)$.

Theorem 4. For every set of norms $N$ and a norm $(a, x)$,

1. $(a, x) \in D_{z}(N)$ iff $x \in O^{z}(N, a)$.

2. $(a, x) \in D_{\text {id }}(N)$ iff $x \in O^{i d}(N, a)$.

3. $(a, x) \in D_{c d}(N)$ iff $x \in O^{c d}(N, a)$.

Proof. The proof is trivial and safely left to the readers.

\section{Cross-stage Rules}

In this section we investigate cross-stage rules, which affects more than one stages. Such rules typically have the form of transitivity. We discuss the following rules:

- plain transitivity (T): from $(a, x)$ and $(x, y)$ to $(a, y)$

- cumulative transitivity (CT): from $(a, x),(a \wedge x, y)$ to $(a, y)$

- mediated cumulative transitivity (MCT): from $\left(a, x^{\prime}\right), x^{\prime} \vdash x$ and $(a \wedge x, y)$ to $(a, y)$

- aggregative cumulative transitivity (ACT): from $(a, x),(a \wedge x, y)$ to $(a, x \wedge y)$

$\mathrm{T}$ is used in the input/output logic for constitutive norms [3]. CT is involved in deriv $_{3}$ and deriv $_{4}$. MCT and ACT are introduced by Stolpe [25] and Parent and van der Torre [23] respectively.

Definition 7. $D_{t}(N)$ is the smallest set of norms such that $N \subseteq D_{t}(N)$ and $D_{t}(N)$ is closed under the Trule.

The corresponding semantics for $D_{t}(N)$ is defined in an inductive manner.

Definition 8. For every set of norms $N$ and formula a, we define $O^{t}(N, a)=\bigcup_{i=1}^{\infty} N_{t}^{i}(\{a\})$. Here for a set $A, N_{t}^{i}(A)$ is defined as follows:

- $N_{t}^{1}(A)=N(A)$

- $N_{t}^{i+1}(A)=N\left(N^{i}(A)\right)$

The semantics defined above is sound and complete with respect to $D_{t}(N)$.

Theorem 5. $(a, x) \in D_{t}(N)$ iff $x \in O^{t}(N, a)$.

\subsection{Fixed point approach}

Concerning other cross-stage rules, on the one hand, it is difficult to define their corresponding semantics. On the other hand, we can use a fixed point approach to define systems containing cross-stage rules together with other rules. We start by giving a fixed point theoretic semantics for out and out $_{3}^{+}$. Then we extend to Stople's mediated reusable input/output logic [25] and Parent and van der Torre's aggregative input/output logic [23]. 


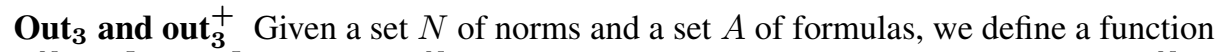
$f_{A}^{N}: 2^{L} \rightarrow 2^{L}$ such that $f_{A}^{N}(X)=C n(A \cup N(X))$. It can be proved that $f_{A}^{N}$ is monotonic with respect to the set theoretical $\subseteq$ relation, and $\left(2^{L}, \subseteq\right)$ is a complete lattice. Then by Tarski's fixed point theorem [27] there exist a least fixed point of $f_{A}^{N}$. The following proposition shows that the least fixed point can be constructed in an inductive manner.

Proposition 1. Let $B_{A}^{N}$ be the least fixed point of the function $f_{A}^{N}$. Then $B_{A}^{N}=\bigcup_{i=0}^{\infty} B_{A, i}^{N}$, where $B_{A, 0}^{N}=C n(A), B_{A, i+1}^{N}=C n\left(A \cup N\left(B_{A, i}^{N}\right)\right)$.

Using the least fixed point, the semantics of out 3 and out ${ }_{3}^{+}$are reformulated as follows:

Theorem 6. For a set of norms $N$ and a formula $a$,

1. $(a, x) \in \operatorname{deriv}_{3}(N)$ iff $x \in C n\left(N\left(B_{a}^{N}\right)\right)$.

2. $(a, x) \in \operatorname{deriv}_{3}^{+}(N)$ iff $x \in C n\left(N_{i d}\left(B_{A}^{N_{i d}}\right)\right)$.

Mediated reusable input/output logic Input/output logic containing the rule of WO is not free from Ross paradox [24]. Stolpe [25] develops the mediated reusable input/output logic such that Ross paradox is avoided without damage the power of WO. Stolpe achieve this by replacing WO and CT in deriv 3 by OEQ and MCT respectively.

Definition 9. (Proof system of mediated reusable input/output logic [25]) $D_{m r}(N)$ is the smallest set of norms such that $N_{z} \subseteq D_{m r}(N)$ and $D_{m r}(N)$ is closed under the following rules: SI, OEQ, AND and MCT.

The semantics of mediated reusable input/output logic is given by an inductive definition.

Definition 10. (Semantics of mediated reusable input/output logic [25]) For every $N \subseteq L \times L, A \subseteq L, x \in O_{m r}(N, A)$ iff $x$ is equivalent to a subset of $\bigcup_{i=0}^{\infty} A_{i}$ where

$$
\begin{aligned}
& \text { - } A_{0}=G(C n(a)), \text { and } \\
& \text { - } A_{n+1}=A_{n} \cup G\left(C n\left(A_{n} \cup\{a\}\right)\right)
\end{aligned}
$$

Theorem 7. (Completeness of mediated reusable input/output logic [25]) $(a, x) \in$ $D_{m r}(N)$ iff $x \in O_{m r}(N, a)$.

Applying the fixed point approach and the previous result about the rule $A N D$, $O E Q$ and $Z$, we have the following equivalence result:

Theorem 8. $(a, x) \in D_{m r}(N)$ iff $x \in C_{a e}\left(N_{z}\left(B_{a}^{N_{z}}\right)\right)$

Proof. Using other results in this paper as a toolbox, the proof is routine. 
Aggregative input/output logic Parent and van der Torre [23] introduce aggregative input/output logic based on the following ideas: on one hand, deontic detachment or cumulative transitivity is fully in line with the tradition of deontic logic. For instance, the Danielsson-Hansson-Lewis semantics $[9,12,17]$ for conditional obligation validates such a law. On the other hand, they also observe that potential counterexamples to deontic detachment may be found in the literature. Parent and van der Torre illustrate this with the following example, due to Broome [5, §7.4]:

You ought to exercise hard everyday

If you exercise hard everyday, you ought to eat heartily

$?^{\star}$ You ought to eat heartily

Intuitively, the obligation to eat heartily no longer holds, if you take no exercise. Like the others, Parent and van der Torre claim that this counterexample suggests an alternative form of detachment, which keeps track of what has been previously detached. They therefore reject the CT rule, and they accept a weaker rule ACT. As a consequence, and following an established tradition in the literature [13,11,28, 25], WO is no longer accepted either.

Definition 11. (Proof system of aggregative input/output logic [23]) $D_{a g}(N)$ is the smallest set of norms such that $N \subseteq D_{a g}(N)$ and $D_{a g}(N)$ is closed under the following rules: SI, OEQ and ACT.

Definition 12. (Semantics of aggregative input/output logic [23]) For every $N \subseteq$ $L \times L, A \subseteq L, x \in O_{a g}(N, A)$ iff there is finite $N^{\prime} \subseteq N$ with $N^{\prime}(A) \neq \emptyset$ such that $\forall B=C n(B)$, if $A \cup N^{\prime}(B) \subseteq B$ then $x \dashv \bigwedge N^{\prime}(B)$.

Parent and van der Torre define $x \in D_{a g}(N, A)$ iff there exist $a_{1}, \ldots, a_{n} \in A$ such that $\left(a_{1} \wedge \ldots \wedge a_{n}, x\right) \in D_{a g}(N)$. The following completeness result is proved [23].

Theorem 9. (Completeness of aggregative input/output logic [23]) Given an arbitrary normative system $N$ and a set $A$ of formulas, $D_{a g}(N, A)=O_{a g}(N, A)$.

Applying the fixed point approach, we reformulate the semantics of aggregative input/output logic as follows:

Theorem 10. $(a, x) \in D_{a g}(N)$ iff there exist finite $N^{\prime} \subseteq N$, such that $N^{\prime}(A) \neq \emptyset$, $x \dashv \Vdash \Lambda N^{\prime}\left(B_{A}^{N^{\prime}}\right)$.

Proof. Having those lemmas on $B_{a}^{N}$ in the appendix, the proof is routine.

\section{Application: input/output logic for constitutive norms}

Constitutive norms are one of the traditional developments of normative reasoning discussed in the handbook of deontic logic. Boella and van der Torre [3] uses a weak input/output logic, decided by rules of IEQ, OEQ, AND and T to reason about constitutive norms. However, we discover the semantics defined in Boella and van der Torre [3] is not sound with respect to the derivation system. In what follows, we first state the mistake of Boella and van der Torre [3], then we use the previous result as a toolbox to build a sound and complete semantics. 
Let $D_{B T}(N)$ be the smallest set of norms such that $N \subseteq D_{B T}(N)$, and $D_{B T}(N)$ is closed under the rules of IEQ, OEQ, AND and T. In Boella and van der Torre [3], the semantics for $D_{B T}(N)$ is defined as follows: given be a set $A$ of formulas, $O(N, A)=$ $\left\{\wedge Y \mid Y \subseteq \bigcup_{i=0}^{\infty} O^{i}(N, A)\right\}$ is calculated as follows, assuming the replacements by logical equivalence:

- $O^{0}(N, A)=\emptyset$

- $O^{i+1}(N, A)=O^{i}(N, A) \cup\left\{y \mid\left(\wedge X^{\prime}, y\right) \in N, X^{\prime} \subseteq O^{i}(N, A)\right\}$.

This semantics is not sound with respect to $D_{B T}(N)$. For an illustration, let $N=$ $\{(p, q)\}$, where $p$ and $q$ are distinct propositional letters. Then $(p, q) \in D_{B T}(N)$. Following the definition of $O(N, A)$, we have $O^{0}(N,\{p\})=\emptyset \cdot O^{1}(N,\{p\})=\emptyset \cup$ $\left\{y \mid\left(\wedge X^{\prime}, y\right) \in N, X^{\prime} \subseteq \emptyset\right\}=\{y \mid(\wedge \emptyset, y) \in N\}=\{y \mid(\top, y) \in N\}=\emptyset$. And similarly, $O^{2}(N,\{p\})=O^{3}(N,\{p\})=\ldots=\emptyset$. Therefore $O(N,\{p\})=\emptyset$ and $q \notin O(N,\{p\})$. This shows that the semantics $O(N, A)$ is not sound for $D_{B T}(N)$. Using the results in this paper, a sound and complete semantics for $D_{B T}(N)$ is defined as follows.

Definition 13. For every set of norms $N$ and formula a, let $O_{B T}(N, a)=\bigcup_{i=1}^{\infty} N_{B T}^{i}(\{a\})$. Here for a set of formulas $A$,

- $N_{B T}^{1}(A)=C_{a e}\left(N\left(C_{e}(A)\right)\right)$

- $N_{B T}^{i+1}(A)=C_{a e}\left(N_{B T}^{i}(A) \cup N\left(N_{B T}^{i}(A)\right)\right)$.

with $C_{a e}(A)=\left\{b \mid \exists a_{1}, \ldots, a_{n} \in A, a_{1} \wedge \ldots \wedge a_{n} \dashv \vdash b\right\}$.

$C_{a e}$, read as "closed under aggregation and equivalence", is a combination of $C_{e}$ defined in Section 3 and $C_{a}$ defined in Section 4. For convenience we will use $N_{B T}^{i}(a)$ to represent $N^{i}(\{a\})$. The following two theorems show that our semantics is sound and complete.

Theorem 11. $(a, x) \in D_{B T}(N)$ iff $x \in O_{B T}(N, a)$.

\section{Related work}

Input/output logic is reformulated by Bochman [2] to model production and causal reasoning. Bochman uses bimodel, which is an order pair of logically closed and consistent set of formulas, to interpret an ordered pair of formulas $(a, x) .{ }^{2}$ A production semantics is a set of bimodels. An ordered pair $(a, x)$ is valid in a production semantics $B$ iff for all $(U, V) \in B$, if $a \in U$ then $x \in V$.

Restrictions are imposed to production semantics. A production semantics $B$ is inclusive if for all $(U, V) \in B, V \subseteq U . B$ is a possible worlds semantics if for all $(U, V) \in B, U, V$ are maximal consistent sets. For a set $N$ of ordered pairs of formulas which contains $(T, \top)$ and $(\perp, \perp)$, Bochman's production semantics is sound and complete for $\operatorname{deriv}_{1}(N$, inclusive production semantics is sound and complete for $\operatorname{deriv}_{3}(N)$ and possible worlds semantics is sound and complete for $\operatorname{deriv}_{2}(N)$.

\footnotetext{
${ }^{2}$ Bochman uses $a \Rightarrow x$ instead of $(a, x) . a \Rightarrow x$ is read as "If $a$ is true, then $x$ is caused".
} 
All of Bochman's production semantics validates at the same time IEQ, OEQ, SI, WO, and AND. Use the technical result of this paper, we can anatomize production semantics. For example, if we define weak bimodel as a consistent set of formulas which is closed under logical equivalence, and a weak production semantics is a set of weak bimodels. Then weak production semantics validates IEQ and OEQ, but neither SI nor WO. Things will get interesting for the weak production semantics which validate cross-stage rules. We leave this as a future work.

\section{Conclusion and future work}

In this paper we anatomize input/output logic. We analyze various derivation rules in isolation and define the corresponding semantics. We thus create a toolbox to build input/output logic. We use this toolbox to correct a mistake appeared in the work of applying input/output logic to constitutive norms. We further develop fixed point characterizations for input/output logics involving rules of cumulative transitivity and present new completeness proofs.

Concerning future works, except the problem mentioned in the end of the related work section, we consider the follows:

- all the input/output logics in this paper are based on propositional logic. Parent et al. [21] build input/output logic on intuitionistic logic. STIT logic is a tool preferred for many deontic logicians $[14,16,4,26]$. It is worthy studying how to build input/output logic based on STIT logic.

- Norms, and more generally conditionals, can be interpreted using neighborhood semantics $[6,15]$. How to compare the operational semantics of this paper to neighborhood semantics?

\section{References}

1. Carlos Alchourron and Eugenio Bulygin. Normative Systems. Springer-Verlag, Wien New York, 1971.

2. Alexander Bochman. A causal approach to nonmonotonic reasoning. Artificial intelligence, 160(1-2):105-143, 2004.

3. Guido Boella and Leendert van der Torre. A logical architecture of a normative system. In Lou Goble and John-Jules Ch. Meyer, editors, Deontic Logic and Artificial Normative Systems, volume 4048 of Lecture Notes in Computer Science, pages 24-35, Utrecht, The Netherlands, 2006. Springer.

4. Jan Broersen. The Encyclopedia of Philosophy and the Social Sciences, chapter Deontic Logic and Agency. SAGE publications, 2013.

5. John Broome. Rationality Through Reasoning. Wiley-Blackwell, West Sussex, UK, 2013.

6. Brian Chellas. Modal logic: an introduction. Cambridge University Press, Cambridge, 1980.

7. Ian Chiswell and Wilfrid Hodges. Mathematical logic. Oxford University Press, 2007.

8. Silvano Colombo Tosatto, Guido Boella, Leendert van der Torre, and Serena Villata. Abstract normative systems: Semantics and proof theory. In Proceedings of the Thirteenth International Conference on Principles of Knowledge Representation and Reasoning, pages 358-368, 2012. 
9. Sven Danielsson. Preference and Obligation: Studies in the Logic of Ethics. Filosofiska freningen, Uppsala, 1968.

10. Dov Gabbay, John Horty, Xavier Parent, Ron van der Meyden, and Leendert van der Torre, editors. Handbook of Deontic Logic and Normative Systems. College Publications, London, 2013.

11. Lou Goble. Murder most gentle: the paradox: deepens. Philosophical Studies, 64:217-227, 1991.

12. Bengt Hansson. An analysis of some deontic logics. Noûs, pages 373-398, 1969.

13. S. O. Hansson. Preference-based deontic logic (PDL). Journal of Philosophical Logic, 19:75-93, 1990.

14. John Horty. Agency and Deontic Logic. Oxford University Press, New York, 2001.

15. Andrew Jones and Marek Sergot. A formal characterization of institutionalised power. Logic journal of the IGPL, 3:427-443, 1996.

16. Barteld Kooi and Allard Tamminga. Moral conflicts between groups of agents. Journal of Philosophical Logic, 37:1-21, 2008.

17. David Lewis. Counterfactuals. Blackwell, Oxford, 1973.

18. David Makinson and Leendert van der Torre. Input-output logics. Journal of Philosophical Logic, 29:383-408, 2000.

19. David Makinson and Leendert van der Torre. Constraints for input/output logics. Journal of Philosophical Logic, 30(2):155-185, 2001.

20. David Makinson and Leendert van der Torre. Permission from an input/output perspective. Journal of Philosophical Logic, 32:391-416, 2003.

21. Xavier Parent, Dov Gabbay, and Leendert van der Torre. An intuitionistic basis for input/output logic. In S.O. Hasson, editor, David Makinson on Classical Methods for NonClassical Problems. Springer, 2012.

22. Xavier Parent and Leendert van der Torre. I/O logic. In John Horty, Dov Gabbay, Xavier Parent, Ron van der Meyden, and Leendert van der Torre, editors, Handbook of Deontic Logic and Normative Systems. College Publications, 2013.

23. Xavier Parent and Leendert van der Torre. Put your parachute on, and jump out! Technical report, 2014. to appear in Proceedings of DEON 2014.

24. Alfred Ross. Imperatives and logic. Theoria, 7(5371), 1941.

25. Audun Stolpe. Normative consequence: The problem of keeping it whilst giving it up. In Ron van der Meyden and Leendert van der Torre, editors, Proceedings of the 9th international conference on Deontic Logic in Computer Science, DEON '08, pages 174-188, Berlin, Heidelberg, 2008. Springer-Verlag.

26. Xin Sun. Conditional ought, a game theoretical perspective. In J. Lang H. van Ditmarsch and S. Ju, editors, Logic, Rationality, and Interaction: Proceedings of the Thire International Workshop, pages 356-369, Guangzhou, China, October 2011.

27. Alfred Tarski. A lattice-theoretical fixpoint theorem and its applications. Pacific Journal of Mathematics, 5(2):285-309, 1955.

28. Leendert van der Torre and Yaohua Tan. Contrary-to-duty reasoning with preference-based dyadic obligations. Ann. Math. Artif. Intell., 27(1-4):49-78, 1999.

29. Georg von Wright. Deontic logic. Mind, 60:1-15, 1952.

\section{Appendix}

\section{Theorem 2}

1. $(a, x) \in D_{i e}(N)$ iff $x \in O^{i e}(N, a)$. 
2. $(a, x) \in D_{s i}(N)$ iff $x \in O^{s i}(N, a)$.

3. $(a, x) \in D_{\text {or }}(N)$ iff $x \in O^{\text {or }}(N, a)$.

Proof. The case for the first two items are easy and left to the reader. Here we focus on the third item.

(left-to-right) Assume $(a, x) \in D_{\text {or }}(N)$, then either $(a, x) \in N$ or $(a, x)$ is derived by the OR rule. The first case is easy to prove. Here we focus on the second case. If $(a, x)$ is derived by the OR rule, then there exist $(b, x) \in D_{o r}(N),(c, x) \in D_{o r}(N)$ and $a$ is $b \vee c$. By induction hypothesis we know $x \in O^{\text {or }}(N, b)$ and $x \in O^{o r}(N, c)$. Now for every $B^{*}$ such that $a \in B^{*}$ and $B^{*}$ is disjunctive, we have $b \vee c \in B^{*}$ since $a$ is $b \vee c$. Note that $B^{*}$ is disjunctive, so we further have either $b \in B^{*}$ or $c \in B^{*}$. If $b \in B^{*}$, then $B^{*}$ is a disjunctive set contains $b$. So we have $x \in O^{\text {or }}(N, b)=\bigcap\{N(B): b \in B, B$ is a disjunctive set $\} \subseteq N\left(B^{*}\right)$. Hence $x \in N\left(B^{*}\right)$. If $c \in B^{*}$, we can similarly deduce $x \in N\left(B^{*}\right)$. Therefore no matter $b \in B^{*}$ or $c \in B^{*}$, we have $x \in N\left(B^{*}\right)$. Therefore $x \in O^{o r}(N, a)$.

(right-to-left) We will give a constructive proof for this theorem. To achieve, we need to introduce the concept of disjunctive parsing tree for formulas. Here we adopt the definition of tree and parsing from Chiswell and Hodges [7].

Definition 14 ([7]). A tree is an ordered pair $(N, D)$ where

(a) $N$ is a finite non-empty set whose elements are called nodes;

(b) D is function that takes each node $\mu \in N$ to a sequence (possibly empty) of distince nodes: $D(\mu)=\left(\nu_{1}, \ldots, \nu_{n}\right)$. The nodes $\nu_{1}, \ldots, \nu_{n}$ are called the daughters of $\mu$, and $\mu$ is called the mother of $\nu_{1}, \ldots, \nu_{n}$.

(c) Every node except one has exactly one mother; the exception is a node called the root.

(d) There are no cycles, that is, sequences $\nu_{1}, \ldots, \nu_{k}(k>1)$, where $\nu_{k}=\nu_{1}$ and each $\nu_{i}$ with $1 \leq i<k$ has mother $\nu_{i+1}$.

Definition 15 ([7]). Given a tree $(N, D)$,

(a) The number of daughters of a node is called its arity.

(b) A node of arity 0 is called a leaf.

(c) A path from node $\nu$ to node $\mu$ is a set of nodes $\left\{\nu_{0}, \ldots, \nu_{k}\right\}$ where $\nu_{0}$ is $\nu, \nu_{k}$ is $\mu$, and fore each $i \leq k, \nu_{i}$ is the mother of $\nu_{i+1}$. A path from the root to a leaf is called a branch.

Now we are ready to defint disjunctive parsing tree for every formula.

Definition 16 (disjunctive parsing tree). Given a formula $\varphi \in L$, the disjunctive parsing tree $P(\varphi)$ is a tree such that:

(a) $\varphi$ is the root of $P(\varphi)$.

(b) Every node which is not a leaf has arity 2.

(c) A node $\psi$ has daughters $\psi_{1}$ and $\psi_{2}$ iff $\psi$ is $\psi_{1} \vee \psi_{2}$.

(d) We define the height for each node as follows: every leaf has height 0 . If $\mu$ is a node with daughters $\nu_{1}, \nu_{2}$, then the height of $\mu$ is $\max \left\{\right.$ height $\left(\nu_{1}\right)$, height $\left.\left(\nu_{2}\right)\right\}+1$. 
Lemma 1. For every formula $\varphi$, every branch of $P(\varphi)$ is a disjunctive set.

Proof. Let $B$ be an arbitrary branch of $P(\varphi)$. For every $\varphi_{1} \vee \varphi_{2} \in B$, we know $\varphi_{1}$ and $\varphi_{2}$ are the only daughters of $\varphi_{1} \vee \varphi_{2}$. Therefore $B$ contains either $\varphi_{1}$ or $\varphi_{2}$. Hence $B$ is disjunctive.

Lemma 2. Let $(a, x)$ be a norm and $N$ a normative system. If for every $B_{i}$ which is a branch of $P(A)$, there exist $a_{i} \in B_{i}$ such that $\left(a_{i}, x\right) \in N$, then $(a, x) \in D_{\text {or }}(N)$.

Proof. Since the length of $a$ is always finite, we know $P(a)$ is also finite. So we assume $\left\{B_{1}, \ldots, B_{n}\right\}$ is the set of all branches of $P(a)$.

Here we just consider the worst case, other cases are easier. In the worst case we have for every $B_{i}$, the element $a_{i} \in B_{i}$ such that $\left(a_{i}, x\right) \in N$ is of height 0 . Then by applying the OR rule finitely many times we know that for every $a_{i}^{\prime} \in B_{i}$ with height $\left(a_{i}^{\prime}\right)=1,\left(a_{i}^{\prime}, x\right) \in D_{\text {or }}(N)$. Similarly we can deduce that for every $a_{i}^{\prime \prime} \in B_{i}$ with height $\left(a_{i}^{\prime \prime}\right)=2,\left(a_{i}^{\prime \prime}, x\right) \in D_{o r}(N)$. This progress can go on and on and we will eventually have $(a, x) \in D_{\text {or }}(N)$ since the height of $a$ is finite.

Now we finish the third item of theorem 2. Suppose $x \in O^{\text {or }}(N, a)$, then $x \in$ $\bigcap\{N(B): a \in B, B$ is a disjunctive set $\}$. Let $\left\{B_{1}, \ldots, B_{n}\right\}$ be the set of all branches of $P(a)$. For every such $B_{i}$ we know $a \in B_{i}$ and $B_{i}$ is disjunctive by Lemma 1 . Therefore we have $x \in N\left(B_{i}\right)$. That is, there exist $a_{i} \in B_{i}$ such that $\left(a_{i}, x\right) \in N$. Now by Lemma 2 we know $(a, x) \in D_{o r}(N)$.

To prove the left to right direction of Theorem 5, we need the following lemmas:

Lemma 3. For all $i \geq 1$, if $A \subseteq B$ the $N_{t}^{i}(A) \subseteq N_{t}^{i}(B)$.

Proof. We prove by induction. If $i=1$, then $N_{t}^{1}(A)=N(A) \subseteq N(B) \subseteq N_{t}^{1}(B)$.

Assume the statement is true for $k$, consider $k+1 . N_{t}^{k+1}(A)=N\left(N_{t}^{k}(A)\right)$, $N_{t}^{k+1}(B)=N\left(N_{t}^{k}(B)\right)$. By induction hypothesis we have $N_{t}^{k}(A) \subseteq N_{t}^{k}(B)$. Therefore $N\left(N_{t}^{k}(A)\right) \subseteq N\left(N_{t}^{k}(B)\right), N_{t}^{k+1}(A) \subseteq N_{t}^{k+1}(B)$.

Lemma 4. For all $i, j \geq 1$, if $x \in N_{t}^{i}(a)$ and $y \in N_{t}^{j}(x)$, then for some $k, y \in N_{t}^{k}(a)$

Proof. Suppose $x \in N_{t}^{i}(a)$ and $y \in N_{t}^{j}(x)$. Let $k=i+j$, then by the above lemma we have $y \in N_{t}^{j}\left(N_{t}^{i}(a)\right)=N_{t}^{i+j}(a)$.

Theorem $5(a, x) \in D_{t}(N)$ iff $x \in O^{t}(N, a)$.

Proof. (left to right) Assume $(a, x) \in D_{t}(N)$, then either $(a, x) \in N$ or $(a, x)$ is derived by the T rule. The first case is easy to prove. Here we just focus on the second case.

Assume $(a, y) \in D_{t}(N)$ and it is deduced by the T rule. Then there exist $(a, x) \in$ $D_{t}(N)$ and $(x, y) \in D_{t}(N)$. By induction hypothesis we have $x \in O^{t}(N, a)$ and $y \in O(N, x)$. That is, $x \in \bigcup_{i=1}^{\infty} N_{t}^{i}(a)$ and $y \in \bigcup_{i=1}^{\infty} N_{t}^{i}(x)$. Therefore there exist some $i, j$ such that $x \in N_{t}^{i}(a)$ and $y \in N_{t}^{j}(x)$. Therefore we have $y \in N_{t}^{i+j}(x)$ by the Lemma 4. Hence $y \in \bigcup_{i=1}^{\infty} N_{t}^{i}(a)$. $(a, y) \in D_{t}(N)$.

(right to left) Assume $x \in O^{t}(N, a)$, then $x \in \bigcup_{i=1}^{\infty} N_{t}^{i}(a)$. Then there exist some $i, x \in N_{t}^{i}(a)$. Now by Lemma 5 below we have $(a, x) \in D_{t}(N)$. 
Lemma 5. For all $i \geq 1$, if $x \in N_{t}^{i}(a)$ then $(a, x) \in D_{t}(N)$.

Proof. We prove by induction. If $i=1$, then from $x \in N_{t}^{1}(a)=N(a)$ we can deduce $(a, x) \in N \subseteq D_{t}(N)$. Now for $i=k+1$, if $x \in N_{t}^{k+1}(a)$, then $x \in N\left(N_{t}^{i}(a)\right)$. Therefore there exist $y \in N_{t}^{i}(a),(y, x) \in N$. By I.H. we have $(a, y) \in D_{t}(N)$ and then use the rule of $\mathrm{T}$ we have $(a, x) \in D_{t}(N)$.

Proposition 1 Let $B_{A}^{N}$ be the least fixed point of the function $f_{A}^{N}$. Then $B_{A}^{N}=\bigcup_{i=0}^{\infty} B_{A, i}^{N}$, where $B_{A, 0}^{N}=C n(A), B_{A, i+1}^{N}=C n\left(A \cup N\left(B_{A, i}^{N}\right)\right)$.

Proof. We first prove that $\bigcup_{i=0}^{\infty} B_{A, i}^{N}$ is a fixed point of $f_{A}^{N}$. We prove by showing the following:

1. $A \subseteq \bigcup_{i=0}^{\infty} B_{A, i}^{N}$ : this is because $A \subseteq C n(A)=B_{A, 0}^{N} \subseteq \bigcup_{i=0}^{\infty} B_{A, i}^{N}$

2. $N\left(\bigcup_{i=0}^{\infty} B_{A, i}^{N}\right) \subseteq \bigcup_{i=0}^{\infty} B_{A, i}^{N}$ : For every $x \in N\left(\bigcup_{i=0}^{\infty} B_{A, i}^{N}\right)$, there exist $k$ such that $x \in N\left(B_{A, k}^{N}\right) \subseteq B_{A, k+1}^{N} \subseteq \bigcup_{i=0}^{\infty} B_{A, i}^{N}$.

3. $C n\left(\bigcup_{i=0}^{\infty} B_{A, i}^{N}\right)=\bigcup_{i=0}^{\infty} B_{A, i}^{N}$ : the right-to-left direction is obvious; for the other direction: assume $x \in C n\left(\bigcup_{i=0}^{\infty} B_{A, i}^{N}\right)$, then there exist $x_{1}, \ldots x_{n} \in \bigcup_{i=0}^{\infty} B_{A, i}^{N}$ such that $x_{1} \wedge \ldots \wedge x_{n} \vdash x$. Therefore there exist $k$ such that $x_{1}, \ldots x_{n} \in B_{A, k}^{N}$. Hence $x \in B_{A, k+1}^{N} \subseteq \bigcup_{i=0}^{\infty} B_{A, i}^{N}$.

With the above four clauses in hand, we can prove that $f_{A}^{N}\left(\bigcup_{i=0}^{\infty} B_{A, i}^{N}\right) \subseteq \bigcup_{i=0}^{\infty} B_{A, i}^{N}$. For the other direction, we prove by induction on $i$ that for every $i, B_{A, i}^{N} \subseteq f_{A}^{N}\left(\bigcup_{i=0}^{\infty} B_{A, i}^{N}\right)$. Here we omit the details.

So we have proved that $\bigcup_{i=0}^{\infty} B_{A, i}^{N}$ is a fixed point of $f_{A}^{N}$. To prove that it is the least fixed point, we can again prove by induction that for every $i, B_{A, i}^{N} \subseteq f_{A}^{N}(B)$, where $B$ is a fixed point of $f_{A}^{N}$. Here we omit the details.

The following lemmas are needed to prove the left to right direction of Theorem 6 .

Lemma 6. For every $A \subseteq L, N \subseteq L \times L, A \subseteq B_{A}^{N}$

Proof. By Lemma 1, the proof is trivial.

Lemma 7. For every $a \in L, N \subseteq L \times L, B_{a}^{N}=C n\left(B_{a}^{N}\right)$. Here $B_{a}^{N}$ is short for $B_{\{a\}}^{N}$.

Proof. By the compactness of propositional logic and Lemma 1, the proof is easy.

Lemma 8. For every $a, b \in L, N \subseteq L \times L$, if $a \vdash b$ then $B_{b}^{N} \subseteq B_{a}^{N}$.

Proof. We will prove that for every $i, B_{b, i}^{N} \subseteq B_{a, i}^{N}$.

We prove by induction on $i$.

If $i=0$, then $B_{b, 0}^{N}=C n(b) \subseteq C n(a) \subseteq B_{a, 0}^{N}$. Assume $i=k+1$ and $B_{b, k}^{N} \subseteq$ $B_{a, k}^{N}$. Then $B_{b, k+1}^{N}=C n\left(\{b\} \cup N\left(B_{b, k}^{N}\right)\right)$. From $B_{b, k}^{N} \subseteq B_{a, k}^{N}$ we deduce $N\left(B_{b, k}^{N}\right) \subseteq$ $N\left(B_{a, k}^{N}\right)$. Now by the monotony of $C n(\bullet)$ we know $C n\left(\{b\} \cup N\left(B_{b, k}^{N}\right)\right) \subseteq C n(\{a\} \cup$ $\left.N\left(B_{a, k}^{N}\right)\right)$. Hence $B_{b, k+1}^{N} \subseteq B_{a, k+1}^{N}$.

So we have proved for every $i, B_{b, i}^{N} \subseteq B_{a, i}^{N}$. With this result in hand, we can easily deduce that $B_{b}^{N} \subseteq B_{a}^{N}$. 
Lemma 9. If $x \in C n\left(N\left(B_{a}^{N}\right)\right)$, then $x \in B_{a}^{N}$.

Proof. By Lemma 1, it is easy to verify that $N\left(B_{a}^{N}\right) \subseteq B_{a}^{N}$ and $C n\left(B_{a}^{N}\right) \subseteq B_{a}^{N}$. The result then follows.

Lemma 10. If $x \in C n\left(N\left(B_{a}^{N}\right)\right)$, then $B_{a}^{N}=B_{a \wedge x}^{N}$.

Proof. It's easy to prove that $B_{a}^{N} \subseteq B_{a \wedge x}^{N}$. For the other direction, we need to prove that for every $i, B_{a \wedge x, i}^{N} \subseteq B_{a}^{N}$. We prove this by induction on $i$.

- Base step: Let $i=0$, we then have $B_{a \wedge x, i}^{N}=C n(a \wedge x)$. By Lemma 6 we have $a \in B_{a}^{N}$. By Lemma 9 we have $x \in B_{a}^{N}$. Then by Lemma 7 we have $a \wedge x \in B_{a}^{N}$. - Inductive step: Assume for $i=k, B_{a \wedge x, k}^{N} \subseteq B_{a}^{N}$. Then $B_{a \wedge x, k+1}^{N}=C n(\{a \wedge$ $\left.x\} \cup N\left(B_{a \wedge x, k}^{N}\right)\right)$. From $B_{a \wedge x, k}^{N} \subseteq B_{a}^{N}$ we know there exist $j$ such that $B_{a \wedge x, k}^{N} \subseteq$ $\bigcup_{i=0}^{j} B_{a, i}^{N}$. Therefore $\left.N\left(B_{a \wedge x, k}^{N}\right)\right) \subseteq N\left(\bigcup_{i=0}^{j} B_{a, i}^{N}\right) \subseteq \bigcup_{i=0}^{j+1} B_{a, i}^{N} \subseteq B_{a}^{N}$. So we have proved $\left.N\left(B_{a \wedge x, k}^{N}\right)\right) \subseteq B_{a}^{N}$. By the base step we have $a \wedge x \in B_{a}^{N}$. Then by Lemma 7 we know $C n\left(\{a \wedge x\} \cup N\left(B_{a \wedge x, k}^{N}\right)\right) \subseteq B_{a}^{N}$. That is, $B_{a \wedge x, k+1}^{N} \subseteq B_{a}^{N}$.

Theorem 6 For a set of norms $N$ and a formula $a$,

1. $(a, x) \in \operatorname{deriv}_{3}(N)$ iff $x \in C n\left(N\left(B_{a}^{N}\right)\right)$.

2. $(a, x) \in \operatorname{deriv}_{3}^{+}(N)$ iff $x \in C n\left(N_{i d}\left(B_{A}^{N_{i d}}\right)\right)$.

Proof. Here we focus on the case for $d_{e r i v}$, the other case is similar. (left to right) Assume $(a, x) \in \operatorname{deriv}_{3}(N)$, we prove by induction on the length of derivation.

- (Base step) Assume $(a, x) \in N$, then by Lemma 6 we have $a \in B_{a}^{N}$. Hence $x \in N\left(B_{a}^{N}\right) \subseteq C n\left(N\left(B_{a}^{N}\right)\right)$.

- Assume $(b, x) \in$ deriv $_{3}$ and it is derived at the last step by using SI from $(a, x) \in$ deriv $_{3}$ and $b \vdash a$. Then by inductive hypothesis we have $x \in C n\left(N\left(B_{a}^{N}\right)\right)$. By Lemma 8 we know $B_{a}^{N} \subseteq B_{b}^{N}$. Therefore we further have $N\left(B_{a}^{N}\right) \subseteq N\left(B_{b}^{N}\right)$, $C n\left(N\left(B_{a}^{N}\right)\right) \subseteq C n\left(N\left(\overline{B_{b}^{N}}\right)\right)$. Hence $x \in C n\left(N\left(B_{b}^{N}\right)\right)$.

- Assume $(a, x \wedge y) \in \operatorname{deriv}_{3}(N)$ and it is derived at the last step by using AND from $(a, x)$ and $(a, y)$. Then by inductive hypothesis we have $x \in C n\left(N\left(B_{a}^{N}\right)\right)$ and $y \in C n\left(N\left(B_{a}^{N}\right)\right)$. Therefore $x \wedge y \in C n\left(N\left(B_{a}^{N}\right)\right)$.

- Assume $(a, y) \in \operatorname{deriv}_{3}(N)$ and it is derived by using WO form $(a, x) \in \operatorname{deriv}_{3}(N)$ and $x \vdash y$. Then by inductive hypothesis we have $x \in C n\left(N\left(B_{a}^{N}\right)\right)$. Since $x \vdash y$, we can prove that $y \in C n\left(N\left(B_{a}^{N}\right)\right)$.

- Assume $(a, y) \in \operatorname{deriv}_{3}(N)$ and it is derived by using CT form $(a, x) \in \operatorname{deriv}_{3}(N)$ and $(a \wedge x, y) \in \operatorname{deriv}_{3}(N)$. Then by inductive hypothesis we have $x \in C n\left(N\left(B_{a}^{N}\right)\right)$ and $y \in C n\left(N\left(B_{a \wedge x}^{N}\right)\right)$. Then by Lemma 10 we have $B_{a}^{N}=B_{a \wedge x}^{N}$. Therefore $y \in C n\left(N\left(B_{a}^{N}\right)\right)$.

(right to left) Assume $x \in C n\left(N\left(B_{a}^{N}\right)\right)$, then there exist $x_{1}, \ldots, x_{n} \in N\left(B_{a}^{N}\right)$ such that $x_{1} \wedge \ldots \wedge x_{n} \vdash x$. For each $i \in\{1, \ldots, n\}$, from $x_{i} \in N\left(B_{a}^{N}\right)$ we know there is $a_{i} \in B_{a}^{N}$ such that $\left(a_{i}, x_{i}\right) \in N$. From $a_{i} \in B_{a}^{N}$ we know there exist $k$ such 
that $a_{i} \in B_{a, k}^{N}$. Now by Lemma 11 we know $\left(a, x_{i}\right) \in \operatorname{deriv}_{3}(N)$. Then applying the AND rule we have $\left(a, x_{1} \wedge \ldots x_{n}\right) \in \operatorname{deriv}_{3}(N)$. Then by the WO rule we have $(a, x) \in \operatorname{deriv}_{3}(N)$.

Lemma 11. For all $i$, if $b \in B_{a, i}^{N}$ and $(b, x) \in N$, then $(a, x) \in \operatorname{deriv}_{3}(N)$

Proof. We prove by induction on $i$.

- Base step: Let $i=0$. Then $b \in B_{a, 0}^{N}=C n(a)$. Hence $a \vdash b$. Therefore we can apply SI to $a \vdash b$ and $(b, x)$ to derive $(a, x)$.

- Inductive step: Assume for $i=k$, if $b \in B_{a, k}^{N}$ and $(b, x) \in N$, then $(a, x) \in$ $\operatorname{deriv}_{3}(N)$. Now let $b \in B_{a, k+1}^{N}$. Then $b \in C n\left(\{a\} \cup N\left(B_{a, k}^{N}\right)\right)$, and there exist $b_{1} \ldots b_{n} \in N\left(B_{a, k}^{N}\right)$ such that $a \wedge b_{1} \wedge \ldots \wedge b_{n} \vdash b$. Then apply SI to $(b, x) \in N$ and $a \wedge b_{1} \wedge \ldots \wedge b_{n} \vdash b$ we have $\left(a \wedge b_{1} \wedge \ldots \wedge b_{n}, x\right) \in \operatorname{deriv}_{3}(N)$. Note that for each $i \in\{1, \ldots, n\}$, from $b_{i} \in N\left(B_{a, k}^{N}\right)$ we know there is $a_{i} \in B_{a, k}^{N}$ such that $\left(a_{i}, b_{i}\right) \in N$. Now by inductive hypothesis we have $\left(a, b_{i}\right) \in \operatorname{deriv}_{3}(N)$. Then applying the AND rule we have $\left(a, b_{1} \wedge \ldots \wedge b_{n}\right) \in \operatorname{deriv}_{3}(N)$. From $\left(a, b_{1} \wedge \ldots \wedge\right.$ $\left.b_{n}\right) \in \operatorname{deriv}_{3}(N)$ and $\left(a \wedge b_{1} \wedge \ldots \wedge b_{n}, x\right) \in D_{3}(N)$ we can adopt the $C$ T rule to derive $(a, x) \in \operatorname{deriv}_{3}(N)$.

To prove the left to right direction of Theorem 11, we need the following lemmas:

Lemma 12. For all $A$, if $i \leq j$ then $N_{B T}^{i}(A) \subseteq N_{B T}^{j}(A)$

Proof. The proof is trivial and left to the readers.

Lemma 13. For all $i \geq 1$, if $A \subseteq B$ the $N_{B T}^{i}(A) \subseteq N_{B T}^{i}(B)$.

Proof. We prove by induction. And we focus on the inductive step. Assume $N_{B T}^{i}(A) \subseteq$ $N_{B T}^{i}(B)$, consider $N_{B T}^{i+1}(A)$ and $N_{B T}^{i+1}(B)$. Note that $N_{B T}^{i+1}(A)=C_{a e}\left(N_{B T}^{i}(A) \cup\right.$ $\left.N\left(N_{B T}^{i}(A)\right)\right)$. By I.H. we have $N_{B T}^{i}(A) \subseteq N_{B T}^{i}(B)$. By the monotonicity of $N(\bullet)$ we have $N\left(N_{B T}^{i}(A)\right) \subseteq N\left(N_{B T}^{i}(B)\right)$. Therefore $N_{B T}^{i}(A) \cup N\left(N_{B T}^{i}(A)\right) \subseteq N_{B T}^{i}(B) \cup$ $N\left(N_{B T}^{i}(B)\right)$. Therefore $C_{a e}\left(N_{B T}^{i}(A) \cup N\left(N_{B T}^{i}(A)\right)\right) \subseteq C_{a e}\left(N_{B T}^{i}(B) \cup N\left(N_{B T}^{i}(B)\right)\right)$ by the monotonicity of $C_{a e}$. That is, $N_{B T}^{i+1}(A) \subseteq N_{B T}^{i+1}(B)$.

Lemma 14. For all $i, j \geq 1$, for all set $A, N_{B T}^{i}\left(N_{B T}^{j}(A)\right) \subseteq N_{B T}^{i+j}(A)$.

Proof. We prove by induction on $i$.

If $i=1$, then $N_{B T}^{1}\left(N_{B T}^{j}(A)\right)=C_{a e}\left(N\left(C_{e}\left(N_{B T}^{j}(A)\right)\right)\right)=C_{a e}\left(N\left(N_{B T}^{j}(A)\right)\right)$. $N_{B T}^{1+j}(A)=C_{a e}\left(N_{B T}^{j}(A) \cup N\left(N_{B T}^{j}(A)\right)\right)$. By monotonicity of $C_{a e}$ we have that $C_{a e}\left(N\left(N_{B T}^{j}(A)\right)\right) \subseteq C_{a e}\left(N_{B T}^{j}(A) \cup N\left(N_{B T}^{j}(A)\right)\right)$. Therefore $N_{B T}^{1}\left(N_{B T}^{j}(A)\right) \subseteq$ $N_{B T}^{1+j}(A)$.

Now for the inductive step. Consider $N_{B T}^{i+1}\left(N_{B T}^{j}(A)\right)$ and $N_{B T}^{i+1+j}(A)$. Note that $N_{B T}^{i+1}\left(N_{B T}^{j}(A)\right)=C_{a e}\left(N_{B T}^{i}\left(N_{B T}^{j}(A)\right) \cup N\left(N_{B T}^{i}\left(N^{j}(A)\right)\right)\right)$. And $N_{B T}^{i+1+j}(A)=$ $C_{a e}\left(N_{B T}^{i+j}(A) \cup N\left(N_{B T}^{i+j}(A)\right)\right)$. By I.H. we have $N_{B T}^{i}\left(N_{B T}^{j}(A)\right) \subseteq N_{B T}^{i+j}(A)$, and by the monotonicity of $N$ we have $N\left(N_{B T}^{i}\left(N_{B T}^{j}(A)\right)\right) \subseteq N\left(N_{B T}^{i+j}(A)\right)$. Then we have $C_{a e}\left(N_{B T}^{i}\left(N_{B T}^{j}(A)\right) \cup N\left(N_{B T}^{i}\left(N_{B T}^{j}(A)\right)\right)\right) \subseteq C_{a e}\left(N_{B T}^{i+j}(A) \cup N\left(N_{B T}^{i+j}(A)\right)\right)$. That is, $N_{B T}^{i+1}\left(N_{B T}^{j}(A)\right) \subseteq N_{B T}^{i+1+j}(A)$. 
Lemma 15. For all $i, j \geq 1$, if $x \in N_{B T}^{i}(a)$ and $y \in N_{B T}^{j}(x)$, then there exist some $k$ such that $y \in N_{B T}^{k}(a)$

Proof. Assume $x \in N_{B T}^{i}(a)$ and $y \in N_{B T}^{j}(x)$, then by Lemma 13 we have $y \in$ $N_{B T}^{j}\left(N_{B T}^{i}(a)\right)$. Now by the lemma above we have $y \in N_{B T}^{i+j}(a)$.

Lemma 16. For all $i \geq 1$, if $x \in N_{B T}^{i}(a)$ and $y \in N_{B T}^{i}(a)$, then $x \wedge y \in N_{B T}^{i}(a)$

Proof. Trivial. Here we skip the details.

To prove the right to left direction of Theorem 11, we need the following lemma.

Lemma 17. For all $i \geq 1$, if $x \in N_{B T}^{i}(a)$ then $(a, x) \in D_{B T}(N)$.

Proof. We prove by induction. If $i=1$, from $x \in N_{B T}^{1}(a)$ we know $x \in C_{a e}\left(N\left(C_{e}(a)\right)\right)$. Therefore there exist $x_{1} \ldots x_{m} \in N(C e(a))$ such that $x \dashv x_{1} \wedge \ldots \wedge x_{m}$. From $x_{1} \ldots x_{m} \in N(C e(a))$ we can deduce that there exist $\left(a_{1}, x_{1}\right), \ldots,\left(a_{n}, x_{m}\right) \in N$ such that $a_{1}, \ldots, a_{m} \in C_{e}(a)$. Therefore $a \dashv a_{1}, \ldots, a \dashv a_{m}$. Now we use IEQ we have $\left(a, x_{1}\right), \ldots,\left(a, x_{m}\right) \in D_{B T}(N)$. And use the AND rule finite times we have $\left(a, x_{1} \wedge \ldots \wedge x_{m}\right) \in D_{B T}(N)$. Then by OEQ we know $(a, x) \in D_{B T}(N)$.

Now for the inductive step. Assume $x \in N_{B T}^{i+1}(a)$,then $x \in C_{a e}\left(N_{B T}^{i}(a) \cup N\left(N_{B T}^{i}(A)\right)\right)$. Therefore there exist $x_{1}, \ldots, x_{m} \in N_{B T}^{i}(a)$ and $y_{1}, \ldots, y_{n} \in N\left(N_{B T}^{i}(a)\right)$ such that $x \dashv x_{1} \wedge \ldots \wedge x_{m} \wedge y_{1} \wedge \ldots \wedge y_{n}$. By I.H. we can deduce $\left(a, x_{1}\right), \ldots,\left(a, x_{m}\right) \in$ $D_{B T}(N)$ from $x_{1}, \ldots, x_{m} \in N_{B T}^{i}(a)$. And from $y_{1}, \ldots, y_{n} \in N\left(N_{B T}^{i}(a)\right)$ know there exist $a_{1}, \ldots, a_{n} \in N_{B T}^{i}(a)$ such that $\left(a_{1}, y_{1}\right), \ldots,\left(a_{n}, y_{n}\right) \in N$. By I.H. we can deduce $\left(a, a_{1}\right), \ldots,\left(a, a_{n}\right) \in D_{B T}(N)$ from $a_{1}, \ldots, a_{n} \in N_{B T}^{i}(a)$. Now by using the $\mathrm{T}$ rule $n$ times we have $\left(a, y_{1}\right), \ldots,\left(a, y_{n}\right) \in D_{B T}(N)$. Then by using the AND rule we have $\left(a, x_{1} \wedge \ldots \wedge x_{m} \wedge y_{1} \wedge \ldots y_{n}\right) \in D_{B T}(N)$. Then use OEQ we have $(a, x) \in D_{B T}(N)$.

Theorem $11(a, x) \in D_{B T}(N)$ iff $x \in O_{B T}(N, a)$.

Proof. (left to right) Assume $(a, x) \in D_{B T}(N)$, then either $(a, x) \in N$, or $(a, x)$ is derived by using at the last step one of the rules IEQ, OEQ, T and AND. Here we only deal with the last two cases. Other cases are easy.

Assume $(a, x) \in D_{B T}(N)$ and it is deduced by the $\mathrm{T}$ rule at the last step. Then there exist $(a, y) \in D_{B T}(N)$ and $(y, x) \in D_{B T}(N)$. By I.H. we have $y \in O_{B T}(N, a)$ and $x \in O_{B T}(N, y)$. That is, $y \in \bigcup_{i=1}^{\infty} N_{B T}^{i}(a)$ and $x \in \bigcup_{i=1}^{\infty} N_{B T}^{i}(y)$. Therefore there exist some $i, j$ such that $y \in N_{B T}^{i}(a)$ and $x \in N_{B T}^{j}(y)$. Therefore we have $x \in N_{B T}^{k}(a)$ for some $k$ by Lemma 15 . Hence $x \in \bigcup_{i=1}^{\infty} N_{B T}^{i}(a)$. , $x \in O_{B T}(N)$.

Assume $(a, x) \in D_{B T}(N)$ and it is deduced by the AND rule at the last step. Then there exist $x_{1}, x_{2}$ such that $x$ is $x_{1} \wedge x_{2}$ and $\left(a, x_{1}\right),\left(a, x_{2}\right) \in D_{B T}(N)$. By I.H. we have $x_{1} \in \bigcup_{i=1}^{\infty} N_{B T}^{i}(a)$ and $x_{2} \in \bigcup_{i=1}^{\infty} N_{B T}^{i}(a)$. Therefore for some $m, n$ we have $x_{1} \in N_{B T}^{m}(a)$ and $x_{2} \in N_{B T}^{n}(a)$. Let $k=\max \{m, n\}$, then by Lemma 12 we have $x_{1}, x_{2} \in N_{B T}^{k}(a)$. Then by Lemma 16 we have $x_{1} \wedge x_{2} \in N_{B T}^{k}(a)$. That is, $x \in N_{B T}^{k}(a), x \in \bigcup_{i=1}^{\infty} N_{B T}^{i}(a)$ and $x \in O_{B T}(N, a)$.

(right to left) Assume $x \in O_{B T}(N, a)$, then $x \in \bigcup_{i=1}^{\infty} N_{B T}^{i}(a)$. Then there exist some $k, x \in N_{B T}^{k}(a)$. Now by Lemma 17 we have $(a, x) \in D_{B T}(N)$. 